\title{
Considering emissions in the transit network design and frequency setting problem with a heterogeneous fleet
}

\author{
Javier Duran-Micco ${ }^{\mathrm{a} *}$, Evert Vermeir $^{\mathrm{a}}$, Pieter Vansteenwegen ${ }^{\mathrm{a}}$ \\ ${ }^{a}$ Mobility Research Center - CIB, KU Leuven, Celestijnenlaan 300, 3001 Leuven, Belgium. \\ *Corresponding author. Email: javier.duranmicco@kuleuven.be
}

\begin{abstract}
Urban transportation contributes significantly to $\mathrm{CO} 2$ emissions. Public transport systems are a good strategy to reduce these, but the emissions generated by public transport vehicles should not be neglected during the design of the service. The Transit Network Design and Frequency Setting Problem (TNDFSP) has usually been addressed considering only the passengers' and the operator's point of view. However, we show it is worthwhile to consider also the emissions already during this planning phase.

This paper proposes a memetic algorithm to address the bi-objective TNDFSP where both the total travel time and the $\mathrm{CO}_{2}$ emissions are minimized. The analysis considers a heterogeneous fleet, meaning that buses of different sizes and technologies can be assigned under a budget constraint. The results on benchmark instances show that the proposed memetic algorithm performs as well as state-of-the-art algorithms where $\mathrm{CO}_{2}$ emissions are not considered. In addition, several experiments are carried out to observe the effect of incorporating emissions and heterogeneous fleet into the model. The heterogeneous fleet allows reducing travel times and emissions at the same time, compared to solutions without a heterogeneous fleet. Moreover, the explicit minimization of $\mathrm{CO}_{2}$ emissions within a bi-objective framework allows illustrating the trade-off between both objectives. Reductions of about $30 \%$ in the emissions can be achieved by increasing the travel time only $1 \%$, while the costs for the operator remain the same. This clearly demonstrates the benefits of considering both the $\mathrm{CO}_{2}$ emissions and a heterogeneous fleet during the design stage of public transport systems.
\end{abstract}

\section{Keywords}

Transportation; Transit Network Design; Frequency Setting; $\mathrm{CO}_{2}$ emissions; Bi-objective memetic algorithm 


\section{Introduction}

Public transport systems are crucial in modern cities since they allow commuters to move in an efficient, sustainable and affordable manner. At the same time, they generate benefits to the rest of the city by reducing congestion in the streets. It is recognized that the design of such systems must take into account the quality of the service provided to the passengers as well as the costs of its operation, two aspects that are in conflict. However, the emissions generated by the public transport systems have typically received much less attention, especially during the early stages of the design of the system. Nevertheless, the man made Greenhouse Gas emissions are indicated as one of the main causes of global warming and therefore there is a worldwide effort to reduce these emissions. As one of the main sources of pollution, cities play a crucial role in these efforts. Consequently, efficient public transport systems should not only reduce emissions by reducing the need for private cars, they should also minimize the pollution generated by their own operations. So, in this paper, we will consider the emissions already early during the design stage in order to obtain public transport systems that offer a high quality service, with reasonable costs and with lower emissions.

The design of a public transport system is typically divided into several sub-problems that span from strategical to tactical and operational decisions. A commonly adopted division consists of: route network design, frequency setting, timetabling, fleet assignment and crew assignment (Ceder and Wilson, 1986). However, there are no unique definitions for each of these problems and, for example, the route network design may or may not include also decisions regarding the infrastructure network and which stops should be served in each line. Since each of the sub-problems is a complex problem by itself, they are usually solved in sequence, with feedback to previous problems when required. However, each of these planning stages has an impact on the other stages, for example, the frequencies have a direct impact on which routes passengers will prefer and on the number of buses needed to operate a certain line. This means it has a significant impact on the network design problem, which is usually solved in advance. Hence, although many papers deal with the network design problem without frequency setting, it is important to consider at least a preliminary frequency setting, which can be adjusted in more detail in a future step (López-Ramos, 2014). Considering network design and frequency setting together is usually denoted as the Transit Network Design and Frequency Setting Problem (TNDFSP) (Farahani et al., 2013; Guihaire and Hao, 2008). Although network design is also called line planning in many other papers (e.g., Canca et al., 2017; Goerigk and Schmidt, 2017; Schöbel and Scholl, 2006) we will use network design and frequency setting in the rest of the paper. The TNDFSP basically consists of designing a set of lines, which are represented as a sequence of nodes on the infrastructure network, and assigning a frequency to each line such that the bus capacity suffices to satisfy the demand. 
In this paper, the TNDFSP, also explicitly considering emissions, is addressed by means of a specifically designed memetic algorithm. Moreover, the effects are analyzed of considering a heterogeneous fleet, consisting of buses with different capacities and emissions. Since this is one of the first studies considering emissions during the TNDFSP, we limit the emissions considered to only $\mathrm{CO}_{2}$ emissions. Other pollutants, such as $\mathrm{NO}_{x}, \mathrm{PM}$ or VOC, can be considered in further studies.

The most important contribution of our work is to illustrate the importance of considering $\mathrm{CO}_{2}$ emissions already during the line planning stage. Therefore, we define and address a bi-objective TNDFSP, minimizing the passenger travel time and $\mathrm{CO}_{2}$ emissions as independent objectives and considering operator costs as a constraint. An alternative would be to use a weighted sum of the passenger travel time and $\mathrm{CO}_{2}$ emissions as a single objective, but then all results would strongly depend on the weights used. Furthermore, the bi-objective approach allows to analyze the trade-off between these two objectives. Our algorithm deals with a heterogeneous fleet so that buses with different sizes and emissions can be considered. Until now, a heterogeneous fleet was only considered in a limited way when allocating a limited number of electric buses within a network. More contributions are present in the methodology applied to solve the problem. In particular, we identify specific strategies that may be useful for future algorithm design for this and similar problems, such as using a different objective function during local search (the proportions of demand covered without transfers instead of minimizing travel time), using only fast local search moves and not "remove" during the local search, performing two evaluation strategies as a way to obtain a more diverse set of solutions in a reasonable computing time and using problem specific cross-over and mutation operators. Additionally, we show that limiting the number of transfers for the passengers to one is a good strategy to reduce substantially the computing times with almost no impact on the quality of the solutions.

Section 2 presents an overview of the related literature. In Section 3, the specific variant of the TNDFSP addressed in this paper is described. Then, the proposed memetic algorithm is explained in Section 4. In Section 5, the experimental results on five benchmark networks are presented and discussed. Finally, conclusions and future work are presented in Section 6.

\section{Literature Review}

There is an increasing amount of research on variants of the TNDFSP and different techniques have been applied to approach these problems. Most studies apply genetic or evolutionary algorithms, in its basic form or in hybrid algorithms, which have demonstrated good behavior in solving the problem (Afandizadeh et al., 2013; Arbex and da Cunha, 2015; Asadi Bagloee and Ceder, 2011; Beltran et al., 2009; Buba and Lee, 2018; Cipriani et al., 2012; Duran et al., 2019; Goerigk and Schmidt, 2017; Liu et al., 2010; Mahdavi Moghaddam et al., 2019; Ngamchai and Lovell, 2003; Owais and Osman, 2018; Pternea et al., 
2015). In particular, Zhao et al. (2015) propose a Memetic Algorithm, although the operators and moves they use differ significantly from the operators we propose in Section 4.2. In addition, other metaheuristics have been used, such as Particle Swarm Optimization (Jha et al., 2019), Adaptive LNS (Canca et al., 2017), GRASP (Mauttone and Urquhart, 2009), Bee Colony Optimization (Nikolić and Teodorović, 2014), Artificial Bee Colony (Szeto and Jiang, 2014) and Intelligent Agent Optimization (Blum and Mathew, 2011). Also, iterative heuristic approaches for this problem have been proposed (Lee and Vuchic, 2005; Yan et al., 2013). Only a few studies have applied exact methods, and given the complexity of the problem, they are applicable only to rather small networks or simplified versions of the problem (Cancela et al., 2015; Schöbel and Scholl, 2006; Wan and Lo, 2009; Zhang et al., 2014). Studies that address the transit network design problem without frequency setting (TNDP) also tend to apply metaheuristics (e.g., Ahmed et al., 2018; Feng et al., 2019; Islam et al., 2019; John et al., 2014; Mumford, 2013; Nayeem et al., 2014). Some of these papers include modifications in traditional algorithms that take into account specific characteristics of the problem. For example, in (Mahdavi Moghaddam et al., 2019) a new operator is applied to the rejected individuals within a genetic algorithm, which is intended to reduce the number of transfers required by passengers. Likewise, in (Islam et al., 2019) a new measure is proposed to compute shortest paths when designing lines, which considers both distance and demand. Moreover, in (Feng et al., 2019; Mumford, 2013; Nayeem et al., 2014), evolutionary algorithm operators have been proposed that consider particular constraints of the TNDP. These techniques served as inspiration for the design of the memetic algorithm described in Section 4.

Although a significant number of papers deals with variants of the TNDFSP, these studies are hard to compare because of the different assumptions and specific characteristics of the problems addressed. For example, some papers consider that all the demand must be served (e.g., Cancela et al., 2015; Mauttone and Urquhart, 2009; Nikolić and Teodorović, 2014) while others set a penalization for the unserved demand (e.g., Arbex and da Cunha, 2015; Buba and Lee, 2018; Pternea et al., 2015). Also, elastic demand may be considered, meaning that the amount of passengers depends on the transit network configuration (e.g., Beltran et al., 2009; Wan and Lo, 2009; Zhang et al., 2014). Most papers assume that the demand is fixed to the bus stops and must be served by buses stopping there, but some papers set the demand to centroids in separated nodes or allow passengers to walk between stations so the access time to the bus stop becomes relevant (e.g., Asadi Bagloee and Ceder, 2011; Liu et al., 2010; Pternea et al., 2015; Szeto and Jiang, 2014). Regarding the configuration of the lines, most authors assume that lines must be served in two directions with the same frequency, but some papers consider also circular lines served in one direction (e.g., Asadi Bagloee and Ceder, 2011; Ngamchai and Lovell, 2003; Zhang et al., 2014). Also, while most papers assume that passengers consider only the travel time for deciding their routes, some papers consider that passengers weigh the time differently when waiting at a stop or travelling inside the bus 
(e.g., Arbex and da Cunha, 2015; Beltran et al., 2009; Cipriani et al., 2012) or that passengers take into account the crowding inside the vehicles (e.g., An and Lo, 2016; Szeto and Jiang, 2014).

A crucial assumption with important implications is related to the transit assignment sub-problem. The transit assignment sub-problem models the expected decisions of the users regarding which lines they will take in a given network to reach their destination. When evaluating the expected passenger travel time of a certain line plan, transit assignment is an unavoidable step. Although mostly only simple models are adopted for the TNDFSP, this is the most time consuming part in the different solution approaches (Farahani et al., 2013). In general, papers that use exact methods adopt system optimal schemes that assume that passengers may be led by the operators to take the routes that minimize an overall objective (e.g., Borndörfer et al., 2007; Cancela et al., 2015). Conversely, most heuristic approaches suppose passengers act selfishly minimizing their own travel time. This is a more realistic assumption but it is more difficult to consider during line planning. The simplest models assume that all passengers take the shortest paths regarding only the in-vehicle travel time (Ngamchai and Lovell, 2003; Zhao et al., 2015). Others consider that the route choice depends also on the frequency, transfers and waiting times. In the last category, a commonly adopted approach is the frequency-share method, which assumes that passengers have a set of attractive lines to reach their destination and they take the first bus that arrives at the stop from any of these lines (Baaj and Mahmassani, 1990, 1991; Blum and Mathew, 2011; Buba and Lee, 2018; Nikolić and Teodorović, 2014). Concerning the transit assignment, we will use the last approach. A relevant characteristic of this assumption is that passengers from the same OD pair may take different paths and that some of these paths can be a little longer than the available shortest path. This method may become more time consuming than simply taking the shortest paths, but it corresponds better to passenger decisions in practice.

The variants of the TNDFSP also differ in the inclusion of several other aspects that attempt to make the models more realistic but that add a considerable amount of complexity to the problem. Some papers consider multi-modal networks where different modes of transportation can interact in several ways, such as in causing congestion or leading to elastic demand (Beltran et al., 2009; Cipriani et al., 2012; Duran et al., 2019; Lee and Vuchic, 2005; Wan and Lo, 2009; Zhang et al., 2014). Other papers tackle the assignment of a limited number of zero emission buses, which have some special requirements in terms of the line length or the terminals that can be used (Beltran et al., 2009; Pternea et al., 2015). Also, stochastic models have been proposed by considering stochastic demand (An and Lo, 2016) or travel times (Yan et al., 2013). Some papers include also decisions related to the infrastructure network such as expansion of streets (Zhang et al., 2014), bus stop locations (Asadi Bagloee and Ceder, 2011; Szeto and Jiang, 2014) or location of depots (Afandizadeh et al., 2013; Szeto and Jiang, 2014). These different assumptions and ways of 
modelling passenger behavior have a great impact on the final complexity of the problem and make it difficult to compare different studies. In Section 3, we will clearly define our problem and discuss in detail the assumptions we will make.

Also many different objectives are considered, typically divided in an user's point of view and an operator's point of view. The user's point of view is generally modelled by the total travel time of all users while the operator's point of view is typically modeled by the number of buses required or the total length of the network. Other metrics that can be used to measure these objectives include the average number of transfers, demand satisfaction, seating capacity and empty seats (UI Abedin Zain et al., 2018). Both points of view are relevant for the design of a transit network, but these are often conflicting. Some studies consider one point of view in the objective and define one or more constraints for the other point of view. In earlier studies, it was common to minimize a simple proxy of the operator's costs as the main objective under the only constraint of serving all demand (Kepaptsoglou and Karlaftis, 2009). More recent approaches use a more complex calculation of the total profit and impose competitive times for served demand compared to a competitive mode (Canca et al., 2017). Other papers take the user's point of view as the most important. The operator costs are then modeled as a constraint on the number of buses (e.g., Buba and Lee, 2018; Szeto and Jiang, 2014; Zhao et al., 2015) or a cost associated to the distance covered by the lines (e.g., Schöbel and Scholl, 2006). Many studies actually consider both objectives in the optimization. In general, both objectives are weighted in one function that represents the social welfare looking for a compromise between them. A few papers consider bi-objective techniques which generate a set of solutions to evaluate the trade-off between both objectives (e.g., Arbex and da Cunha, 2015; Blum and Mathew, 2011; Goerigk and Schmidt, 2017; Mauttone and Urquhart, 2009; Nikolić and Teodorović, 2014). This seems to be the best option in order to make a correct decision considering both points of view, but in general, it is much more difficult to solve. Furthermore, a few papers include externalities, such as environmental impacts, in a function that represents the social welfare (e.g., Beltran et al., 2009; Pternea et al., 2015) but this approach does not allow to explore the trade-off between the different components included in the objective function. In (Jha et al., 2019), a multi-objective approach is proposed considering both user and operator costs. $\mathrm{CO}_{2}$ emissions are considered as a tax within the operator costs, but no analysis on the effect of incorporating this tax is presented. In (Duran et al., 2019) the emissions are explicitly minimized in a multi-objective approach, giving a first insight in the importance of considering emissions during the transit network design stage. However, it considers elastic demand within a multimodal network and it does not consider a heterogeneous fleet. Furthermore, it performs experiments only on small networks. 


\section{Problem statement}

The public transport service is designed for a fixed infrastructure network $G(V, E)$, where the nodes, denoted by $i, j \in V$, represent the stops and the bidirectional edges, denoted by $(i, j) \in E$, the connections between the stops. A distance $c_{i j}$, expressed in minutes, is assigned to each edge. The problem considers a fixed demand between each pair of nodes given by an OD matrix where $d_{i j}$ indicates the passengers per hour that travel between stops $i$ and $j$. Additionally, different types of buses are considered, which vary according to both size and technology, such that each type of bus $b \in B$ has associated a unit cost $p_{b}$, a capacity $q_{b}$ expressed in number of passengers, and a $\mathrm{CO}_{2}$ emission factor $e_{b}$ expressed in grams of $\mathrm{CO}_{2}$ per second. We consider that there is an unlimited number of vehicles of each type of bus available and that all the buses of a single line must be of the same type. These simplifications are made considering that the TNDFSP with homogeneous fleet is already very time-consuming to solve. For example, there is still no optimal solution found for the most used benchmark instance with only 15 nodes and 20 links (Mandl, 1979) when six lines can be operated, even without considering the frequency setting or a heterogeneous fleet.

The problem in this paper is (1) to determine the configuration of a set of lines such that all the demand is covered with at most one transfer, (2) to assign a frequency $f_{l}$ to each line / such that the capacity of the buses is sufficient to transport all the passengers and (3) to assign a type of bus to each line. We limit the problem to covering demand with at most one transfer in order to reduce the required computation time, as have been done in previous studies (e.g., Buba and Lee, 2018; Nikolić and Teodorović, 2014; Pternea et al., 2015). Modifying the problem and solution method to deal with two (or more) transfers is straightforward, but it makes the solution methods much slower and it hardly improves the solutions for the benchmark instances considered. This is illustrated at the end of Section 5.1.

The lines, and the edges, are bidirectional and cannot visit the same node more than once. There are a minimum and a maximum length given for the lines. It should be noted that we do not assume a limited set of feasible lines to be given, as is done in many other papers (e.g., Afandizadeh et al., 2013; Arbex and da Cunha, 2015; Cancela et al., 2015; Goerigk and Schmidt, 2017; Liu et al., 2010; Schöbel and Scholl, 2006; Yan et al., 2013). Any line respecting the abovementioned constraints can be part of the solution. From the operator's point of view, there is a budget constraint limiting the number of buses that can be operated in the system. The number of buses required by each line is calculated such that the amount of vehicles is enough to serve the frequency given the roundtrip length of the line and considering a fixed average speed. The two objectives, considered in a bi-objective framework, are to minimize the total travel time of the passengers, which consist of the in-vehicle travel time, waiting time and a penalization 
for transfers, and to minimize the $\mathrm{CO}_{2}$ emissions generated by the system, which depends directly on the number of buses of each type that is operated. No crowding issues are considered.

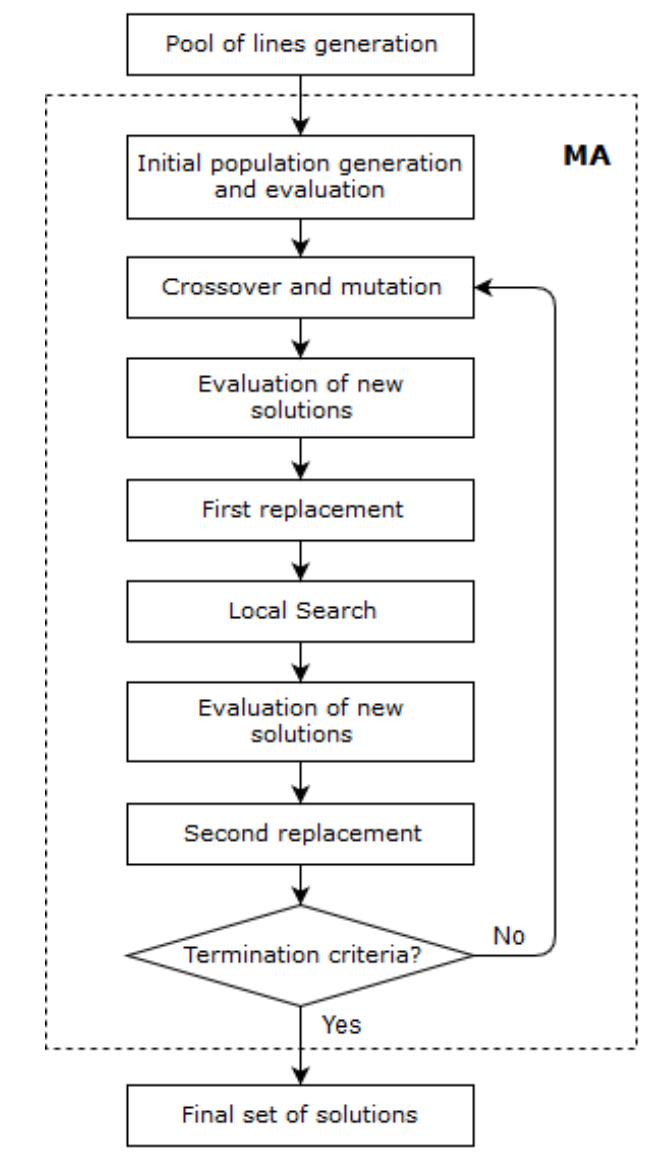

Figure 1: Flowchart of the bi-objective memetic algorithm.

\section{Solution method}

The problem is addressed by means of a memetic algorithm (MA). This technique, first introduced by (Moscato, 1989), is a combination of an evolutionary algorithm, which is suitable for multi-objective problems given its capacity to efficiently explore large areas of the solution space, with a local search technique to intensify the search for high quality solutions. This local search is necessary since the evaluation of every solution is time consuming so the evolutionary algorithm alone might have difficulties to find high quality solutions. The proposed memetic algorithm is based on the NSGA-II framework, which is one of the most popular evolutionary multi-objective algorithms (Talbi, 2009). The basic concept of NSGA-II is that it generates a population of solutions which is improved during successive iterations by means of crossover operators, which combine pairs of existing solutions, and a mutation operator, which randomly modifies individual solutions. In order to deal with two (or more) objectives in the NSGA-II, the 
solutions are ranked according to two criteria. First, solutions are ranked according to the non-dominance ranking, which assigns the highest score to a solution that is not dominated by others. Then, solutions with the same non-dominance ranking are ordered according to the crowding distance. The crowding distance is a measure of how different a solution is compared to its neighboring solutions and is calculated as the circumference of the rectangle defined by the two closest neighbors of a solution. It gives an estimation of the density of surrounding solutions and is required to obtain diverse populations (Deb et al., 2002; Talbi, 2009). Obviously, a specific representation and crossover and mutation operators are designed for solving our TNDFSP. In our MA the solution is represented by a list of lines, each of them with a frequency and a bus type assigned. Figure 1 displays the outline of the proposed algorithm, whose different parts are explained in Sections 4.1, 4.2 and 4.3.

The general framework of the proposed algorithm is that the MA operators (crossover, mutation and local search) deal mainly with the configuration of the lines while the final frequency and bus type assigned to each line are determined during each evaluation procedure. The evaluation procedure also deals with the transit assignment sub-problem. This is very expensive computationally, but it is necessary in order to calculate the value of our two objective functions.

\subsection{MA operators}

The crossover operator is based on (Mumford, 2013) and it generates an offspring from two parents taking around half of the lines from each parent. The offspring is constructed by alternatingly taking lines from each parent. In order to try to obtain feasible solutions that are fully connected, the lines are selected from the parents according to the product between the proportion of nodes absent in the offspring currently under construction and the number of passengers assigned to that line in the corresponding parent. The frequency and the bus type of every line are maintained as in the parent from which the line is taken. This step is repeated until the number of offspring generated is equal to the size of the population.

The mutation is based on the procedure described in (Nayeem et al., 2014). It is applied to a random line and can consist of a small or a big modification. The small modification is to add or remove a terminal node from the line while the big modification is to remove the line and replace it with a new one (taken from the pool of lines used to generate the initial population) but maintaining one of the terminal nodes. Besides the mutation on the line, the bus type assigned to each line is also randomly modified.

Also a repair strategy is included, in case that the network obtained by the crossover or mutation does not cover all the nodes. It consists of searching the shortest extension, from any line, to the unconnected nodes. If it is not possible to repair the solution using this method then the solution is discarded. However, 
the fact that all nodes are covered does not ensure that the network is feasible, since it is not checked that every OD pair is connected with at most one transfer. It is not checked since this is considerably more expensive to evaluate. If it turns out later that the network is infeasible, the solution is discarded then. Although the crossover and mutation operators tend to preserve the shortest paths from the initial pool of lines, through the successive deletions in the mutation and the repair strategy any node in any line might be removed at some point in time and also lines with detours compared to the k-shortest paths may be generated.

Next, the newly generated solutions are evaluated (see Section 4.3). Preliminary experiments showed that the final solution in the evaluation procedure is sensitive to the initial bus size assigned to each line. It was found that the MA generates better results and in a reasonable computing time when for each line plan the evaluation procedure is performed two times. The first time, the initial bus types (size and technology) are inherited from the parent solutions used in the crossover. The second time, the initial bus types are set to small buses on all the lines while the technology is inherited from the parent solutions. The second configuration tends to generate solutions with higher frequencies and lower total travel time for the passengers, but larger emissions. If the two resulting solutions are not dominated, then both are considered in the selection process to generate the new population. Therefore, by evaluating the solutions two times, a better balance is achieved between solutions that minimize the total travel time and the emissions.

After the evaluation, a new population is generated of the same size as the original. This new population is obtained using a replacement strategy that selects the best solutions from both parents and offspring, according to the non-dominance ranking and the crowding distance. To avoid that the population remains the same as the original one, a minimum number of new solutions (offspring) is enforced. This is called "First replacement" in Figure 1.

Once the new population is generated, the local search procedure is applied to a randomly selected subset of the most recent offspring in the population. However, since the evaluation step is computationally expensive and the local search procedure requires many evaluations, it does not minimize the travel time or the emissions directly. Instead of that, the proportion of trips connected without transfers is maximized. This is much faster to compute and update and, in general, solutions with low travel time tend to present high proportions of directly connected OD pairs. Also, for simplicity, the only move considered is to add single nodes to the extreme of the lines. The nodes are added one by one, to both extremes of the line and checking each time that the maximum length and the budget are not surpassed. Each time a line is enlarged, the cost is updated assuming the frequencies remain the same. The advantage is that adding nodes in this way always generates feasible solutions, which is not the case when removing or 
changing nodes. To further speed up the process, the first improvement approach is used, so as soon as an improvement is found it is implemented. For this, the lines are sorted according to their frequencies, starting the search with the line with the lowest frequency. This order is used to attempt to balance the frequencies by enlarging the flows in the least used lines. The search process continues until no more improvements are found. Since a different objective is used, the new solution could improve both, one or none of the original objectives. Thus, the solutions obtained at the end of the local search procedure are fully evaluated (Section 4.3) in order to determine if they should be included in the new population using the same replacement strategy as before. This is called "Second replacement" in Figure 1.

\subsection{Evaluation procedure}

To evaluate the travel time and the emissions generated by the buses it is necessary to solve the transit assignment sub-problem. In this paper, the transit assignment is modelled by a heuristic based on the frequency-share method (Baaj and Mahmassani, 1990). This assumes that the passengers try to minimize their travel time by selecting a set of attractive paths with at most one transfer, before departure. A path is attractive if it is within a certain tolerance compared to the shortest path (in this paper $10 \%$ is used). This way a set $L_{A B}^{i}$ is defined, which contains the potential lines for a passenger in node $i$ that is traveling from $A$ to $B$. This set is defined, and is different for the origin node and every potential transfer node. Once at the bus stop in node $i$, the passenger travelling from $A$ to $B$ takes the first bus that arrives from any of the potential lines of set $L_{A B}^{i}$. The heuristic used is based on (Nikolić and Teodorović, 2014). This evaluation procedure is an iterative process that in addition to the determination of the flows of passengers improves the frequency setting and we also include the assignment of bus types to each line. The process starts with a given set of lines and initial frequencies and bus types assigned to each line. In every iteration, the flows of passengers are calculated (according to the current frequencies) and then the frequencies are updated using equation 1. It considers the flow of passengers in the link with the highest flow and divides it by the capacity of the bus assigned to the line, such that the frequency is enough to transport all the passengers on that line.

$$
f_{l}=\frac{v_{\max }^{l}}{\sum_{b \in B} y_{b}^{l} * q_{b}}
$$

Where:

$f_{l}=$ frequency of line $l$ (buses/hour).

$v_{\text {max }}^{l}=$ maximum flow of passengers on line $l$ (pass/hour).

$y_{b}^{l}=1$, if bus of type $b$ is assigned to line $l$ (only one type of bus per line).

$q_{b}=$ capacity of bus of type $b$ (pass/bus). 
During this process, the size of the buses is also modified following simple rules, such that if a line has a high frequency and small buses then larger buses are assigned, and conversely, if a line has a low frequency and large buses then smaller buses are assigned. For the determination of low or high frequencies, predetermined minimum and maximum frequencies are considered, although the last one is not strictly enforced. The values adopted in this study are 1 and 30 buses/hour respectively. Note that changes on the size of the buses have a direct impact on the frequency setting, according to equation 1.

Once the frequencies and bus types assigned converge, the technology of the buses assigned is modified such that, if there is budget available, cleaner (and more expensive) buses are included, while, if the budget is surpassed, more pollutant (and cheaper) buses are included. The cost of the system depends on the number of buses of each type required to maintain the frequencies on each line, given by equation 2 .

$$
n_{b}^{l}=y_{b}^{l} * \frac{r_{l} * f_{l}}{60}
$$

Where:

$$
\begin{aligned}
& n_{b}^{l}=\text { number of buses of type } b \text { required by line } l . \\
& y_{b}^{l}=\text { binary variable that is } 1, \text { if bus of type } b \text { is assigned to line } l \text { (only one type of bus per line). } \\
& r_{l}=\text { round-trip length of line } l \text { (minutes). } \\
& f_{l}=\text { frequency of line } l \text { (buses/hour). }
\end{aligned}
$$

Then, the total operator's cost of the system is given by equation 3, which depends on the number and type of buses assigned to each line. This value is used in the budget constraint representing the operator's point of view.

$$
O C=\sum_{l \in L} \sum_{b \in B}\left(p_{b} * n_{b}^{l}\right)
$$

Where:

$$
\begin{aligned}
& \text { OC }=\text { operator's cost. } \\
& p_{b}=\text { unit cost of bus type } b . \\
& n_{b}^{l}=\text { number of buses of type } b \text { required by line } l . \\
& L=\text { set of lines in the solution. }
\end{aligned}
$$

Finally, if the solution includes lines with terminal nodes that are not used by any passenger, those nodes are removed from the respective line in order to get a smaller transit network and reduce the number of buses required. If the process converges to a solution where the budget is surpassed (even with the cheapest buses on every line) the solution is maintained but with a high penalization. The procedure does not ensure the frequencies to converge, but the results in this and previous studies show that in most 
cases it does converge in only a few iterations. Solutions where not all the OD pairs are connected with at most one transfer or that do not converge in a given number of iterations are discarded as infeasible solutions.

Once the transit assignment is completed, the value of both objectives can be computed. On the one hand, the total travel time consists of the sum over all passengers of the in-vehicle travel time, waiting time and a penalty for the transfers, as expressed in equation 4. Since for each OD-pair multiple paths are considered and a passenger takes the first bus that reaches the bus stop, both the in-vehicle travel times and the waiting times depend on the set of potential lines, from the attractive paths. When there is a transfer, the waiting time is calculated in the origin and in the transfer point. Furthermore, the transfer penalization is considered by adding a fixed amount in time that is set to 5 minutes in this paper, as in most papers (e.g., Buba and Lee, 2018; Chakroborty and Wivedi, 2002; Fan et al., 2009; John et al., 2014; Mumford, 2013; Nayeem et al., 2014; Nikolić and Teodorović, 2014; Yan et al., 2013).

$$
t_{t t}=t_{i v}+t_{w}+t_{p}
$$

Where:

$t_{t t}=$ total travel time of one passenger (minutes).

$t_{i v}=$ in-vehicle travel time of one passenger (minutes).

$t_{w}=$ waiting time of one passenger, summed over the origin and a potential transfer point (minutes).

$t_{p}=$ penalization time for transfers of one passenger (minutes).

On the other hand, the $\mathrm{CO}_{2}$ emissions depend on the number of buses of each type required, according to equation 5. It gives the system's total emissions, which is just the sum of the whole life-cycle emissions of the buses required by the system.

$$
E M=\sum_{l \in L} \sum_{b \in B}\left(e_{b} * n_{b}^{l}\right)
$$

Where:

$$
\begin{aligned}
& \mathrm{EM}=\text { total } \mathrm{CO}_{2} \text { emissions }\left(\mathrm{t}-\mathrm{CO}_{2}\right) . \\
& e_{b}=\text { emission factor of bus type } b\left(\mathrm{t}_{-} \mathrm{CO}_{2}\right) . \\
& n_{b}^{l}=\text { number of buses of type } b \text { required by line } l .
\end{aligned}
$$

In order to analyze the importance of considering emissions during the transit network design stage, a well-performing algorithm is required. Therefore, several existing techniques, which have been successful to address the TNDFSP, were taken from literature and modified: the evolutionary framework, the specific operators for the problem and the evaluation procedure. Moreover, crucial parts were newly developed 
or specifically modified in order to obtain an efficient algorithm, for example, the initial population generation, the mutation operator, the local search step or the bus type setting during the evaluation procedure. The parameters of the MA were not extensively optimized, but set based on a number of preliminary experiments. For each of the experiments described in Section 5 the algorithm was run for 250 iterations with a population of 30 individuals. The mutation probability was set to 0.05 and the probability of performing a big modification during the mutation procedure to 0.4 . Furthermore, the probability of performing the local search procedure to a newly generated offspring in a new population is 0.75 . Given the stochastic nature of the algorithm, the algorithm was run 5 times for each scenario. As we will show in the next section, the resulting algorithm performs at least as good as state-of-the-art algorithms for the TNDFSP that do not consider $\mathrm{CO}_{2}$ emissions and it addresses effectively and efficiently the bi-objective TNDFSP with $\mathrm{CO}_{2}$ emissions.

\section{Experimental results}

The algorithm was tested on five benchmark networks from the state of the art. The main characteristics of these networks are presented in Table 1. Each of these networks has an associated cost on the edges and an OD matrix, but different instances can be created per network by changing problem parameters such as the characteristics of the bus fleet, the maximum number of lines, the budget constraint, etc. Mandl's network is a 15-node network proposed in 1979 and based on a set of Swiss cities (Mandl, 1979). Although it might seem small, it appears to be difficult to solve and has been used as the most common benchmark for the transit network design problem (e.g., Buba and Lee, 2018; John et al., 2014; Nikolić and Teodorović, 2014; Owais and Osman, 2018). Mumford's networks were proposed more recently and span from 30 to 127 nodes (Mumford, 2013). The demand of the Mumford's networks was originally set on a daily basis and for this study that demand was considered to be equally spread over a period of 16 hours.

Table 1: Networks' characteristics.

\begin{tabular}{cccc}
\hline Network name & Number of nodes & Number of links & $\begin{array}{c}\text { Number of non-zero } \\
\text { OD pairs }\end{array}$ \\
\hline Mandl & 15 & 20 & 172 \\
Mumford0 & 30 & 90 & 870 \\
Mumford1 & 70 & 210 & 4830 \\
Mumford2 & 110 & 385 & 11990 \\
Mumford3 & 127 & 425 & 16002 \\
\hline
\end{tabular}

All the experiments were performed on an Intel ${ }^{\circledR}$ Core $^{\mathrm{TM}}$ i5-7500 CPU $3.40 \mathrm{GHz}$ machine with $8.00 \mathrm{~GB}$ RAM. The MA was coded in C++ and compiled with Visual Studio 2017. Detailed data of the instances and 
details on the solutions from all the experiments performed are available here: https://www.mech.kuleuven.be/en/cib/lp/.

\subsection{Comparison with state of the art}

A first set of experiments was performed to compare the performance of our MA with algorithms from the state of the art. However, the TNDFSP has many different definitions and assumptions and none of the previous studies has tackled the exact same problem as we do, so straightforward comparisons are impossible. Therefore, two types of comparisons are presented. The first one evaluates the performance of our algorithm on the basic network design problem, without considering frequencies. The second one considers network design and frequency setting and allows to evaluate our frequency setting method.

Firstly, our MA is compared with studies that address the basic transit network design problem without considering frequencies. In this case, previous studies are available that consider both Mandl's network and the larger Mumford's networks. In order to make a fair comparison, the MA was slightly adapted to solve this simpler version of the TNDP, which differs from the problem described in Section 3 in several aspects. Firstly, since frequencies are not considered, waiting times and bus capacity are not included in the problem. Secondly, only the travel time is minimized (no emissions are considered) and a homogeneous fleet is considered. Thirdly, the length of the lines is constrained according to the number of nodes instead of the cost of the links. Furthermore, a relevant difference is that previous papers used different assumptions in the transit assignment sub-problem, in the way that all passengers always take the shortest path and can take at most two transfers. Therefore, we modified the evaluation procedure in our algorithm so that passengers always take the shortest path but we still limit the number of transfers to one, given the high computation time that considering more transfers would require for larger networks. However, for the larger instances our solutions are re-evaluated to estimate the impact of allowing or not more than one transfer.

Table 2 and Table 3 show the results of this comparison, for Mandl's and Mumford's networks respectively. Six recent studies are considered in this comparison (Ahmed et al., 2018; Islam et al., 2019; John et al., 2014; Kechagiopoulos and Beligiannis, 2014; Mumford, 2013; Nayeem et al., 2014). We selected these papers because they solve the network design problem with the same characteristics as we do for Mandl's and Mumford's networks and because they provide enough information about their assumptions and solutions to perform a fair comparison. For Mandl, we present the results for instances with the number of lines equal to 4, 6, 7 and 8 , as in the other studies considered. These are the metrics reported for each solution: the average travel time, considering in-vehicle and transfer penalization time $\left(\right.$ ATT'); the proportion of passengers travelling with zero $\left(d_{0}\right)$, one $\left(d_{1}\right)$ or two $\left(d_{2}\right)$ transfer: the proportion 
of unsatisfied demand $\left(\mathrm{d}_{\mathrm{un}}\right)$; and the total length of the lines. However, not all the metrics are presented in every previous study (indicated by “-"). The MA was run 5 times for each network and the best solution found was selected. The solution MA1 is the result obtained from the MA execution and therefore allows at most one transfer. On the other hand, MA2 is obtained after re-evaluating the network determined by MA1, but during the evaluation, the passengers are now allowed to take up to 2 transfers.

Table 2: Comparison of MA performance on Mandl's network without frequencies.

\begin{tabular}{|c|c|c|c|c|c|c|c|c|c|}
\hline Network & Nr. Lines & $\begin{array}{l}\text { Nodes } \\
\text { per line }\end{array}$ & Solution* & $\begin{array}{c}\text { ATT' } \\
\text { (min/pass) }\end{array}$ & $\begin{array}{l}\text { do } \\
(\%)\end{array}$ & $\begin{array}{l}d_{1} \\
(\%)\end{array}$ & $\begin{array}{c}d_{2} \\
(\%)\end{array}$ & $\begin{array}{l}d_{\text {un }} \\
(\%)\end{array}$ & $\begin{array}{l}\text { length } \\
\text { (min) }\end{array}$ \\
\hline \multirow[t]{6}{*}{ Mandl } & 4 & $2-8$ & Mumford & 10.57 & 90.4 & 9.6 & 0.0 & 0.0 & 149 \\
\hline & & & Nayeem** & 10.35 & 95.8 & 3.6 & 0.6 & 0.0 & - \\
\hline & & & Ahmed & 10.48 & 91.8 & 8.2 & 0.0 & 0.0 & 148 \\
\hline & & & K\&B & 10.64 & 91.8 & 7.6 & 0.5 & 0.0 & 150 \\
\hline & & & Islam & 10.51 & 92.4 & 6.8 & 0.8 & 0.0 & - \\
\hline & & & MA1 & 10.50 & 91.5 & 8.5 & 0.0 & 0.0 & 140 \\
\hline \multirow[t]{7}{*}{ Mandl } & 6 & $2-8$ & Mumford & 10.27 & 95.4 & 4.5 & 0.1 & 0.0 & 221 \\
\hline & & & Nayeem** & 10.10 & 98.9 & 1.1 & 0.0 & 0.0 & - \\
\hline & & & John & 10.25 & - & - & - & - & 212 \\
\hline & & & Ahmed & 10.18 & 97.2 & 2.8 & 0.0 & 0.0 & 212 \\
\hline & & & $K \& B$ & 10.23 & 96.2 & 3.5 & 0.3 & 0.0 & 203 \\
\hline & & & Islam & 10.18 & 97.2 & 2.8 & 0.0 & 0.0 & - \\
\hline & & & MA1 & 10.22 & 96.6 & 3.4 & 0.0 & 0.0 & 214 \\
\hline \multirow[t]{6}{*}{ Mandl } & 7 & $2-8$ & Mumford & 10.22 & 96.5 & 3.3 & 0.2 & 0.0 & 264 \\
\hline & & & Nayeem $* *$ & 10.07 & 99.6 & 0.4 & 0.0 & 0.0 & - \\
\hline & & & Ahmed & 10.10 & 98.8 & 1.2 & 0.0 & 0.0 & 250 \\
\hline & & & $\mathrm{K} \& \mathrm{~B}$ & 10.16 & 97.2 & 2.8 & 0.0 & 0.0 & 234 \\
\hline & & & Islam & 10.12 & 98.5 & 1.5 & 0.0 & 0.0 & - \\
\hline & & & MA1 & 10.15 & 98.0 & 2.0 & 0.0 & 0.0 & 231 \\
\hline \multirow[t]{6}{*}{ Mandl } & 8 & $2-8$ & Mumford & 10.17 & 97.6 & 2.3 & 0.1 & 0.0 & 291 \\
\hline & & & Nayeem** & 10.04 & 99.9 & 0.1 & 0.0 & 0.0 & - \\
\hline & & & Ahmed & 10.08 & 99.2 & 0.8 & 0.0 & 0.0 & 272 \\
\hline & & & $K \& B$ & 10.13 & 97.8 & 2.3 & 0.0 & 0.0 & 283 \\
\hline & & & Islam & 10.07 & 99.2 & 0.8 & 0.0 & 0.0 & - \\
\hline & & & MA1 & 10.10 & 98.8 & 1.2 & 0.0 & 0.0 & 254 \\
\hline
\end{tabular}

*Mumford: (Mumford, 2013); Nayeem: (Nayeem et al., 2014); John: (John et al., 2014); Ahmed: (Ahmed et al., 2018); Islam: (Islam et al., 2019); K\&B: (Kechagiopoulos and Beligiannis, 2014)

**No maximum number of nodes per lines is considered.

As shown in Table 2 and Table 3, the MA obtains relatively good solutions in the five instances, being slightly outperformed only by algorithms recently proposed in (Ahmed et al., 2018; Islam et al., 2019). It may also be noted that in general the MA generates solutions with larger networks (last column), although they always satisfy the length constraints. For the purpose of this paper these results are certainly sufficient, since the MA was not designed specifically to solve this problem, but a more extended problem 
with frequency setting and emissions. Table 3 also shows that the ATT' of MA1 is only slightly improved when re-evaluating the solutions to allow two transfers (MA2). Moreover, the results reported in (Ahmed et al., 2018) also present a low proportion (below 1\%) of trips with two transfers. It suggests that although it is desirable to represent passenger behavior realistically by allowing multiple transfers, limiting the number of transfers to one is a good strategy to reduce the computing time of the algorithm. Preliminary experiments showed that solving Mumford3 with our algorithm considering 2 transfers increases the computing time up to 100 times, while the potential improvement in the solutions is very limited.

Table 3: Comparison of MA performance on Mumford's networks without frequencies.

\begin{tabular}{|c|c|c|c|c|c|c|c|c|c|}
\hline Network & Nr. Lines & $\begin{array}{l}\text { Nodes } \\
\text { per line }\end{array}$ & Solution* & $\begin{array}{c}\text { ATT' }^{\prime} \\
\text { (min/pass) }\end{array}$ & $\begin{array}{c}d_{0} \\
(\%)\end{array}$ & $\begin{array}{c}d_{1} \\
(\%)\end{array}$ & $\begin{array}{c}d_{2} \\
(\%)\end{array}$ & $\begin{array}{l}d_{\text {un }} \\
(\%)\end{array}$ & $\begin{array}{l}\text { length } \\
\text { (min) }\end{array}$ \\
\hline \multirow[t]{5}{*}{ Mumford0 } & 12 & $2-15$ & Mumford & 16.05 & 63.2 & 35.8 & 1.0 & 0.0 & 759 \\
\hline & & & John & 15.40 & - & - & - & - & 747 \\
\hline & & & Ahmed & 14.09 & 88.7 & 11.3 & 0.0 & 0.0 & 722 \\
\hline & & & MA1 & 14.88 & 78.0 & 22.0 & 0.0 & 0.0 & 709 \\
\hline & & & MA2 & 14.88 & 78.0 & 22.0 & 0.0 & 0.0 & 709 \\
\hline \multirow[t]{7}{*}{ Mumford1 } & 15 & $10-30$ & Mumford & 24.79 & 36.6 & 52.4 & 10.7 & 0.3 & 2038 \\
\hline & & & Nayeem & 23.96 & 37.7 & 56.4 & 5.9 & 0.0 & - \\
\hline & & & John & 23.91 & - & - & - & - & 1861 \\
\hline & & & Ahmed & 21.69 & 65.7 & 34.2 & 0.1 & 0.0 & 1956 \\
\hline & & & Islam & 22.11 & 55.1 & 43.7 & 1.2 & 0.0 & - \\
\hline & & & MA1 & 22.65 & 53.8 & 46.2 & 0.0 & 0.0 & 2003 \\
\hline & & & MA2 & 22.62 & 53.7 & 45.5 & 0.8 & 0.0 & 2003 \\
\hline \multirow[t]{7}{*}{ Mumford2 } & 56 & $10-22$ & Mumford & 28.65 & 30.9 & 51.3 & 16.4 & 1.4 & 5632 \\
\hline & & & Nayeem & 26.63 & 32.5 & 63.5 & 3.9 & 0.0 & - \\
\hline & & & John & 27.02 & - & - & - & - & 5461 \\
\hline & & & Ahmed & 25.19 & 56.7 & 43.3 & 0.1 & 0.0 & 5257 \\
\hline & & & Islam & 25.08 & 51.9 & 48.0 & 0.2 & 0.0 & - \\
\hline & & & MA1 & 25.46 & 51.1 & 48.9 & 0.0 & 0.0 & 5655 \\
\hline & & & MA2 & 25.46 & 51.1 & 48.5 & 0.4 & 0.0 & 5655 \\
\hline \multirow[t]{7}{*}{ Mumford3 } & 60 & $12-25$ & Mumford & 31.44 & 27.5 & 51.0 & 18.8 & 2.8 & 3665 \\
\hline & & & Nayeem & 29.65 & 29.2 & 64.3 & 6.5 & 0.0 & - \\
\hline & & & John & 29.50 & - & - & - & - & 6320 \\
\hline & & & Ahmed & 28.05 & 50.4 & 48.8 & 0.8 & 0.0 & 6119 \\
\hline & & & Islam & 27.64 & 50.8 & 48.9 & 0.4 & 0.0 & - \\
\hline & & & MA1 & 28.05 & 48.1 & 51.9 & 0.0 & 0.0 & 6809 \\
\hline & & & MA2 & 28.03 & 48.1 & 51.0 & 0.9 & 0.0 & 6809 \\
\hline
\end{tabular}

*Mumford: (Mumford, 2013); Nayeem: (Nayeem et al., 2014); John: (John et al., 2014); Ahmed: (Ahmed et al., 2018); Islam: (Islam et al., 2019)

Secondly, our MA is compared with previous studies that address the TNDP with frequency setting. However, results for the TNDFS don't consider $\mathrm{CO}_{2}$ emissions or heterogeneous fleet and are only available for Mandl's network. The results presented correspond to instances with 6 and 7 lines, which 
are the best results reported in the previous studies. However, a comparison of the results for 4,8 and 12 lines are made available here: https://www.mech.kuleuven.be/en/cib/lp/.

This comparison is presented in Table 4, where for each solution the following metrics are reported: the average travel time, considering in-vehicle, waiting and transfer penalization time (ATT); the proportion of passengers travelling with zero $\left(d_{0}\right)$ and one $\left(d_{1}\right)$ transfer; the fleet size; and the average frequency of the lines (f). Two previous studies are considered, which minimize the total travel time and use a transit assignment method that is equivalent to the one described in Section 4.3. For this comparison, our algorithm was executed 5 times and the solutions with the lowest travel time were selected. The bus capacity is set to 50 passengers per bus, in concordance to the previous studies. The results show that the MA outperforms the previous approaches, finding solutions with lower travel time and a larger proportion of passengers connected without transfers. In addition, the fleet size and frequencies are in the same range as in the previous studies, illustrating that the way the MA determines the frequencies successfully minimizes the average travel time of the passengers.

Table 4: Comparison of MA performance on Mandl's network considering frequencies.

\begin{tabular}{|c|c|c|c|c|c|c|}
\hline Nr. Lines & Solution* & $\begin{array}{c}\text { ATT } \\
\text { (min/pass) }\end{array}$ & $\begin{array}{l}d_{0} \\
(\%)\end{array}$ & $\begin{array}{c}d_{1} \\
(\%)\end{array}$ & $\begin{array}{l}\text { Fleet } \\
\text { size }\end{array}$ & $\begin{array}{c}\mathbf{f} \\
\text { (bus/hour) }\end{array}$ \\
\hline \multirow[t]{3}{*}{6} & Nikolić (users) & 11.90 & 94.34 & 5.66 & 99 & 15.3 \\
\hline & Buba & 12.27 & 96.92 & 3.08 & 92 & 12.8 \\
\hline & $M A$ & 11.74 & 97.17 & 2.83 & 96 & 15.3 \\
\hline \multirow[t]{3}{*}{7} & Nikolić (users) & 11.91 & 94.41 & 5.59 & 99 & 13.1 \\
\hline & Buba & 12.10 & 96.34 & 3.66 & 90 & 9.7 \\
\hline & $M A$ & 11.78 & 96.53 & 3.47 & 98 & 10.3 \\
\hline
\end{tabular}

Based on the comparison above, we conclude that our algorithm performs very well, with solutions better or very close to the ones from the state of the art. Furthermore, the solutions are obtained in a reasonable computing time. The average CPU time to perform one run of the MA is around 5 seconds for Mandl's network and 30, 180, 630 and 1200 seconds respectively for the Mumford's networks. Most of the aforementioned studies don't mention computing times. Ahmed et al. (2018) reported the solution obtained after one run on every network, with computing times that range from 2.5 hours for Mumford0 to 10 hours for Mumford3. However, they also reported slightly worse results obtained with computing times reduced by a factor of ten on each instance. In (John et al., 2014) it is noted that the computing times range from a couple of seconds for Mandl's network up to two days for Mumford3. 


\subsection{Impact of considering $\mathrm{CO}_{2}$ emissions}

Now that we know that our algorithm performs well, a second set of experiments can be performed to assess the impact of considering $\mathrm{CO}_{2}$ emissions and a heterogeneous fleet during the TNDFSP. For these experiments the networks Mumford2 and Mumford3 are not considered because of the higher computing time required to solve them, even more when a heterogeneous fleet and frequency setting needs to be considered. Furthermore, the size of Mumford1 is enough to represent real life instances and hence it is possible to get valuable conclusions from it. The characteristics considered for the different types of buses are displayed in Table 5, where three sizes (small, medium and large) and three technologies (tec1, tec2 and tec3) are considered. The values of cost and the emission factor for each bus type were roughly estimated based on the relative variations of the life cycle costs and emissions observed in the literature (Ercan et al., 2015; Kay et al., 2011). Also the different capacities are roughly based on buses from practice. It should be noted that generic technologies are considered and that in practice some type of buses may impose additional constraints and decisions that are out of scope for this paper. For example, issues related to the consideration of electric buses during the TNDP are addressed and discussed in (Häll et al., 2019; Iliopoulou et al., 2019). For each network, four scenarios are tested by changing the number of available types of buses. Details can be found in Table 6, where the buses included in each scenario are displayed. Scenario $1 \times 1$ is the base scenario with only one type of bus available, while scenario $3 \times 3$ considers nine types of buses. In the scenarios where only one size is available, the medium bus size is used. When only one technology is available it is tec1. As a result, the base scenario is when only medium buses with tec1 are available.

Table 5: Characteristics of the bus types.

\begin{tabular}{cccccc}
\hline Bus id & Size & Technology & $\begin{array}{c}\text { Cost } \\
(-)\end{array}$ & $\begin{array}{c}\text { Capacity } \\
\text { (pass) }\end{array}$ & $\begin{array}{c}\text { Emission factor } \\
\text { (t_CO } \mathbf{~} \text { ) }\end{array}$ \\
\hline 1 & Small & tec1 & 7.5 & 35 & 1394 \\
2 & Medium & tec1 & 10 & 50 & 2024 \\
3 & Large & tec1 & 11.5 & 65 & 2647 \\
4 & Small & tec2 & 8.3 & 35 & 1142 \\
5 & Medium & tec2 & 11 & 50 & 1626 \\
6 & Large & tec2 & 12.7 & 65 & 2111 \\
7 & Small & tec3 & 9.8 & 35 & 709 \\
8 & Medium & tec3 & 13 & 50 & 1021 \\
9 & Large & tec3 & 15 & 65 & 1315 \\
\hline
\end{tabular}

For the comparison and given the bi-objective nature of the problem, after each run two solutions from the final population are considered: the one with the lowest travel time (best-TT) and the one with the least $\mathrm{CO}_{2}$ emissions (best-EM). Table 7 displays, for each network and each scenario, the average values (over 5 runs) of the objective functions (TT and EM) and the total operator's cost (OC) of these two 
solutions, best-TT and best-EM. It should be noted that the operator cost was limited to 1000, 2000 and 16000 and the number of lines was fixed to 6, 10 and 15, for Mandl, Mumford0 and Mumford1 respectively. These values are set in such a way in order to generate meaningful benchmark instances for these experiments. For clarity, also the percentage variation of the travel time and the $\mathrm{CO}_{2}$ emissions compared to the base case are presented between brackets.

Table 6: Description of the scenarios tested.

\begin{tabular}{cccc}
\hline Scenario & Nr. of sizes & Nr. of technologies & $\begin{array}{c}\text { Buses available } \\
\text { (bus id) }\end{array}$ \\
\hline $1 \times 1$ & 1 & 1 & 2 \\
$1 \times 3$ & 1 & 3 & $2,5,8$ \\
$3 \times 1$ & 3 & 1 & $1,2,3$ \\
$3 \times 3$ & 3 & 3 & $1-9$ \\
\hline
\end{tabular}

Table 7: Scenario comparison in networks Mandl, Mumford0 and Mumford1.

\begin{tabular}{|c|c|c|c|c|c|c|c|}
\hline \multirow[b]{2}{*}{ Network } & \multirow{2}{*}{$\begin{array}{c}\text { Scenario } \\
\text { (sizes } x \text { tech) }\end{array}$} & \multicolumn{3}{|c|}{ Best-TT solution } & \multicolumn{3}{|c|}{ Best-EM solution } \\
\hline & & $\begin{array}{c}\text { ATT } \\
\text { (min/pass) }\end{array}$ & $\begin{array}{c}\mathrm{EM} \\
\left(\mathrm{kt} \_\mathrm{CO}_{2}\right) \\
\end{array}$ & $\begin{array}{l}\text { OC } \\
(-)\end{array}$ & $\begin{array}{c}\text { ATT } \\
\text { (min/pass) }\end{array}$ & $\begin{array}{c}\text { EM } \\
\left(\mathrm{kt} \_\mathrm{CO}_{2}\right) \\
\end{array}$ & $\begin{array}{l}\text { OC } \\
(-) \\
\end{array}$ \\
\hline \multirow[t]{4}{*}{ Mandl } & $1 \times 1$ & 11.66 & 196 & 990 & 12.32 & 137 & 698 \\
\hline & $3 \times 1$ & $11.34(-2.8 \%)$ & $182(-7.3 \%)$ & 975 & $11.86(-3.8 \%)$ & $137(-0.3 \%)$ & 725 \\
\hline & $1 \times 3$ & $11.66(0.0 \%)$ & $185(-5.6 \%)$ & 999 & $12.31(-0.1 \%)$ & $70(-49.0 \%)$ & 907 \\
\hline & $3 \times 3$ & $11.38(-2.4 \%)$ & $166(-15.6 \%)$ & 997 & $11.88(-3.6 \%)$ & $69(-49.7 \%)$ & 965 \\
\hline \multirow[t]{4}{*}{ Mumford0 } & $1 \times 1$ & 18.07 & 395 & 1985 & 18.96 & 314 & 1588 \\
\hline & $3 \times 1$ & $17.40(-3.7 \%)$ & 364 (-7.9\%) & 1950 & $18.16(-4.2 \%)$ & $308(-2.0 \%)$ & 1665 \\
\hline & $1 \times 3$ & 18.09 (0.1\%) & $386(-2.2 \%)$ & 1997 & $19.20(1.3 \%)$ & $175(-44.1 \%)$ & 1972 \\
\hline & $3 \times 3$ & $17.52(-3.1 \%)$ & 332 (-15.9\%) & 1998 & 18.97 (0.1\%) & $151(-52.0 \%)$ & 1958 \\
\hline \multirow[t]{4}{*}{ Mumford1 } & $1 \times 1$ & 24.71 & 3099 & 15478 & 25.61 & 2808 & 14078 \\
\hline & $3 \times 1$ & $24.58(-0.5 \%)$ & $3027(-2.3 \%)$ & 15750 & $25.56(-0.2 \%)$ & $2804(-0.2 \%)$ & 14477 \\
\hline & $1 \times 3$ & $24.67(-0.2 \%)$ & $3046(-1.7 \%)$ & 15960 & $25.95(1.3 \%)$ & $2165(-22.9 \%)$ & 15963 \\
\hline & $3 \times 3$ & $24.47(-1.0 \%)$ & $3036(-2.0 \%)$ & 15974 & $25.60(0.0 \%)$ & $1507(-46.4 \%)$ & 15975 \\
\hline
\end{tabular}

The results show that the heterogeneous fleet can have an impact on both objectives, although the impact is much larger for the $\mathrm{CO}_{2}$ emissions. Also, as expected, the impact on the travel time is mainly due to the size of the buses while the impact on the emissions is related more with the technologies. However, the combined effect generates greater benefits. In particular, Table 7 shows that in Mandl's network a reduction of $2.4 \%$ in the travel time or a reduction of $49.7 \%$ in the $\mathrm{CO}_{2}$ emissions can be achieved with the heterogeneous fleet (3x3). Similarly, in Mumford0 the potential improvement achieved is $3.1 \%$ in the travel time and $52.0 \%$ in the $\mathrm{CO}_{2}$ emissions. The impact in Mumford1 is a little smaller, although still the potential reduction is $1.0 \%$ in the travel time and $46.4 \%$ in the $\mathrm{CO}_{2}$ emissions. It is important to note that in the case of the best-TT solutions, the scenario $3 \times 3$ generates solutions that improve both objectives compared to the base scenario $(1 \times 1)$, not only the travel time, while maintaining a constant cost. For 
example, in Table 7, it can be seen that in Mandl's network the scenario with a heterogeneous fleet ( $3 \times 3)$ gets a best-TT solution where the travel time is reduced $2.4 \%$ while the emissions also decrease $15.6 \%$. Another interesting finding, which is less obvious, is that the scenario with only multiple sizes ( $3 \times 1)$ also improves both objectives in the best-TT solution. Based on these results we can conclude that the consideration of a heterogeneous fleet has a significant impact on the solutions obtained. In particular, the consideration of different sizes of buses allows to slightly reduce the total travel time and emissions while the different technologies obviously have a bigger impact on the $\mathrm{CO}_{2}$ emissions. The effect on the total travel time of allowing different sizes can be explained by the opportunity to operate smaller buses with higher frequencies and thus shorter waiting times.

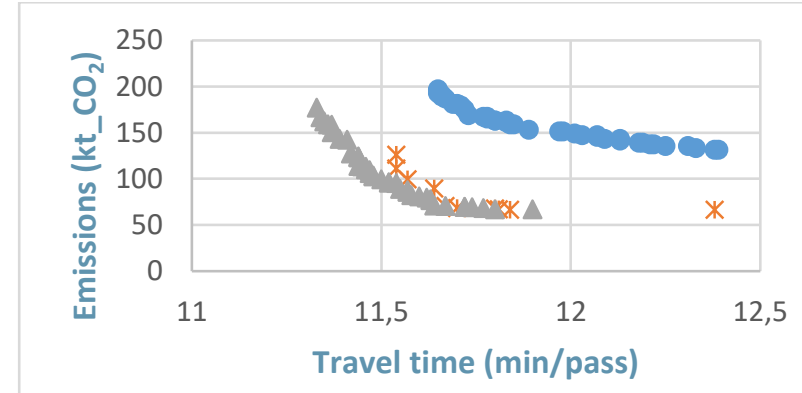

(a) Mandl's network.

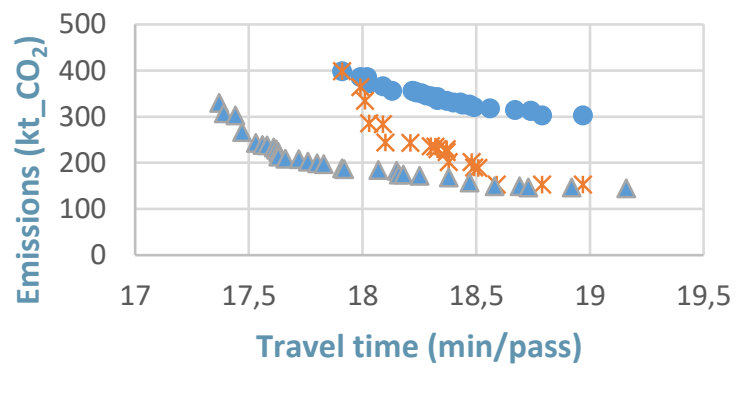

(b) Network Mumford0.

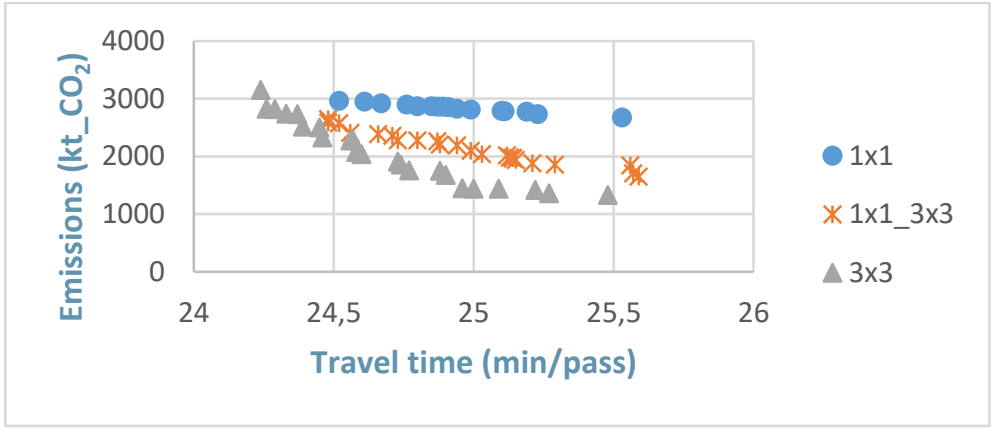

(c) Network Mumford1.

Figure 2: Non dominated solutions found by MA in ten runs, considering homogeneous or heterogeneous fleet during network design.

It must be highlighted that for each network the budget was the same in all scenarios but the total cost of the solutions is not always the same. The cost of the solutions best-TT is generally very close to the budget, meaning that all the resources are used in order to have more buses to reduce the travel time. However, in the scenarios $1 \times 1$ and $3 \times 1$ the solutions best-EM tend to have a smaller cost since when only one technology is available, the number of buses must be reduced in order to reduce emissions. When cleaner technologies are available, more resources are used to use these technologies. 
In some cases, it can be observed that some solutions become slightly worse than in the base scenario. This should not occur since extra opportunities are provided in these scenarios. However, this can be explained by the probabilistic nature of the MA and by the fact that the same number of iterations is used for all the scenarios. Since with more bus alternatives the solution space is much larger, it becomes more difficult for the MA to find better solutions.

Further experiments show the benefits of considering a heterogeneous fleet during the network design stage rather than assigning the buses once the lines are already defined. To illustrate this, the networks of bus lines obtained from the homogenous fleet (scenario 1x1) were re-evaluated operating a heterogeneous fleet on these lines. Figure 2 displays the non-dominated solutions obtained after 10 executions of the MA, for the scenarios with homogeneous fleet $(1 \times 1)$, heterogeneous fleet $(3 \times 3)$ and the re-evaluated solutions from the first case $\left(1 \times 1 \_3 \times 3\right)$. It can be clearly seen that when the heterogeneous fleet is considered during the network design stage, better solutions are generated and a smoother Pareto frontier is obtained.
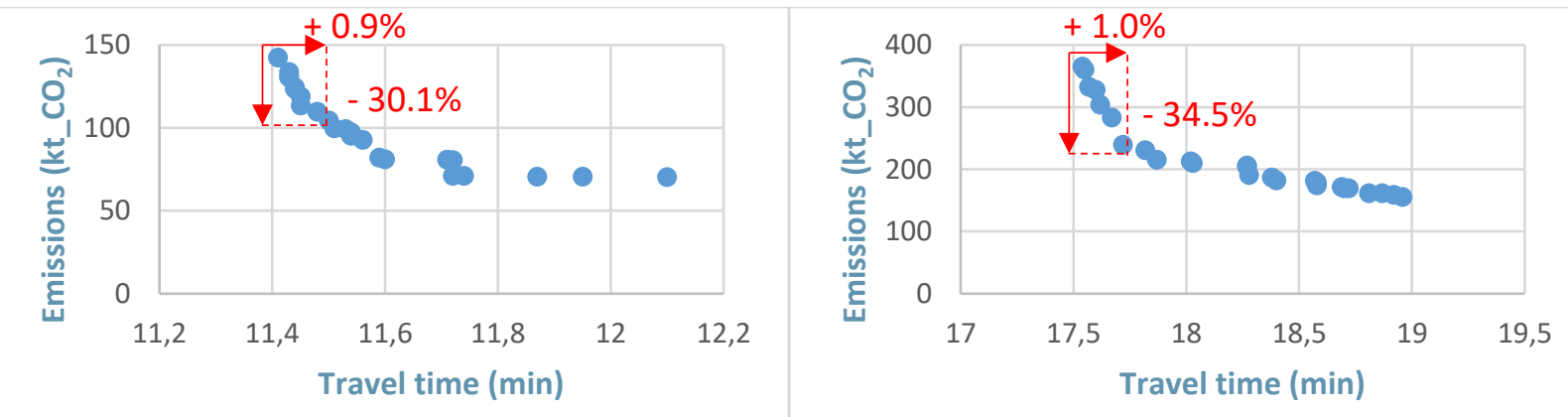

(a) Scenario 3×3 Mandl's network.

(b) Scenario 3x3 network Mumford0.

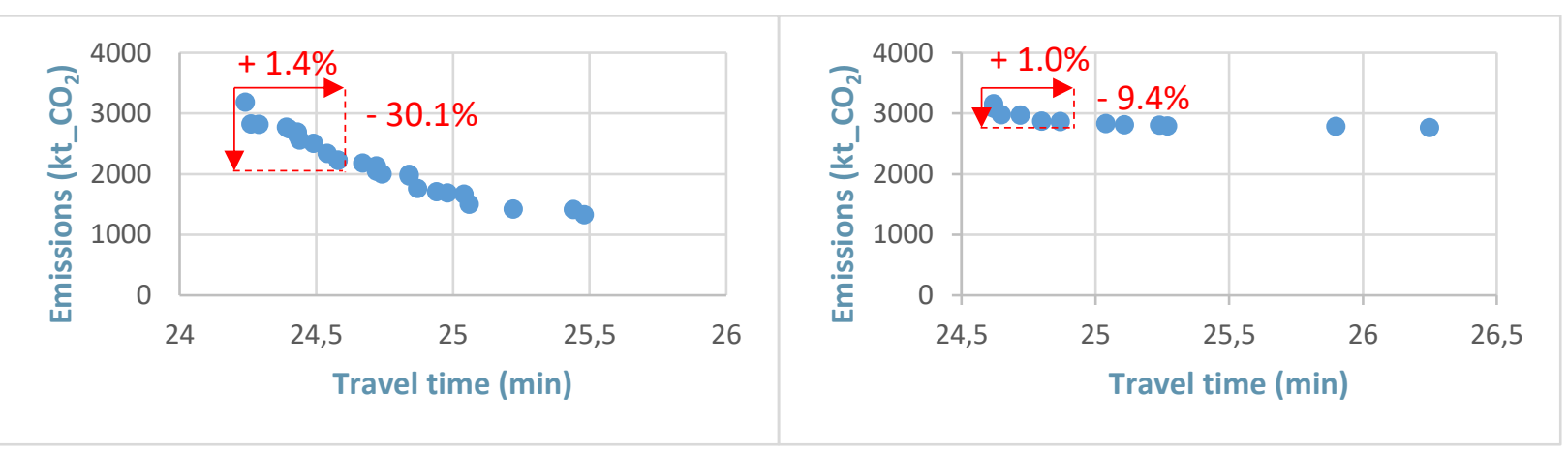

(c) Scenario $3 \times 3$ network Mumford1.

(d) Scenario 1x1 network Mumford1.

Figure 3: Non dominated solutions found by MA in one run.

Clearly, considering $\mathrm{CO}_{2}$ emissions during transit network design and frequency setting is very relevant. Large reductions in the $\mathrm{CO}_{2}$ emissions can be achieved with a relatively small impact on the travel time. 
Figure 3 shows sets of non-dominated solutions found in one run for each network under the scenario $3 \times 3$. Additionally, a set of non-dominated solutions found in the Mumford1 network under the scenario $1 \times 1$ is presented. The upper-left solution in each plot can be considered as the base solution, since it is the one that minimizes the travel time, which is the traditional objective in the literature. It can be seen that from that solution the emissions can be reduced significantly by only slightly increasing the travel time. For example, in Mandl's network the emissions can be reduced by $30 \%$ with an increase of less than $1 \%$ in travel time, compared to the base solution. In the case of network Mumford1, also reductions of around $30 \%$ on the emissions can be achieved by increasing the travel time only around $1.5 \%$. Furthermore, when minimizing only the travel time, the solution obtained would be similar to the base solution, but probably with even larger emissions, since emissions are not considered during the optimization process. Thus, the possible improvements over existing solutions in the literature would be even greater. It is interesting to see in Figure 3 (d) that even in the scenario without a heterogeneous fleet $(1 \times 1)$ the impact is clearly visible. In the network Mumford1, emissions can be reduced by $9.4 \%$ with an increase on the travel time of only $1.0 \%$. In the last case, the reduction of emissions is directly related to a reduction in the number of buses and hence in the total cost. On the other hand, in scenario $3 \times 3$ the total cost is almost the same for all the non-dominated solutions displayed. From these results, we conclude that it is important and interesting to include the $\mathrm{CO}_{2}$ emissions as an objective since it offers a number of opportunities to drastically reduce $\mathrm{CO}_{2}$ emissions while only slightly increasing the travel time and maintaining the operator's cost.

The MA obtains solutions in a reasonable computing time. For the scenario $3 \times 3$, the average CPU time of one run is 6, 70 and 550 seconds respectively for Mandl's network, Mumford0 and Mumford1. These times are higher than the ones reported in Section 5.1, which is not a surprise since the problem solved is more complex by the inclusion of the frequency setting and the bus type assignment. Although the full experiments were not performed on Mumford3, a preliminary experiment showed that the CPU time to solve the $3 \times 3$ scenario was around 2.5 hours. These computing times are certainly acceptable for this kind of problem in practice (but not practical for the repeated calculations in the experimental setup mentioned above). We conclude that the proposed algorithm seems practical to be applied on larger instances as well. It should also be noted that a network of 70 nodes, such as Mumford1, already has the size of smaller real life problems.

\subsection{Detailed analysis of the $3 \times 3$ scenario}

The solutions along the Pareto frontiers tend to present some common characteristics that are similar in the three networks addressed. Table 8 displays more detailed information about a set of non-dominated solutions of one run for the network Mumford1 displayed in Figure 3 (c) (Scenario 3×3). For each solution 
the values of the objective functions ( $A T T$ and EM) are presented, the operator's cost (OC), the number of buses of each type required (categorized according to technology and size), the total number of buses (TB), the total length of the lines (TL), the proportion of passengers that travel without transfers $\left(\mathrm{d}_{0}\right)$, the proportion of passengers that travel with one transfer $\left(d_{1}\right)$ and the average frequency of the lines (f). The operator's cost remains constant and very close to the budget over all solutions which means that the algorithm found solutions that use the available resources. Also, it can be observed that the solutions with lower travel time tend to present a larger proportion of the demand served without transfers and larger lines. On the contrary, the solutions with lower emissions tend to have shorter lines maintaining constant or even reducing the frequencies, so fewer buses are necessary, but cleaner (and more expensive) technologies can be used. As expected, the solutions with lower emissions tend to have more buses with cleaner technologies, which is true for the three networks considered. However, the assignment of bus sizes differs between the networks. In the network Mumford1 (in Table 8) mostly medium and large buses are selected while in Mandl's network mostly small buses are selected. This probably depends on other characteristics of the network, such as the level of demand, the average distances and the budget available. Further research on these aspects could be valuable to improve the bus selection procedure.

Table 8: Non dominated solutions found in one run. Scenario 3×3 network Mumford1.

\begin{tabular}{|c|c|c|c|c|c|c|c|c|c|c|c|c|c|c|c|c|}
\hline \multirow{3}{*}{$\begin{array}{l}\text { sol } \\
\text { id }\end{array}$} & \multirow{3}{*}{$\begin{array}{c}\text { ATT } \\
\text { (min/pass) }\end{array}$} & \multirow{3}{*}{$\begin{array}{c}\mathrm{Em} \\
\left(\mathrm{kt} \_\mathrm{CO}_{2}\right)\end{array}$} & \multirow{3}{*}{$\begin{array}{l}\text { OC } \\
(-)\end{array}$} & $\begin{array}{c}\text { bus } \\
1\end{array}$ & $\begin{array}{c}\text { bus } \\
2\end{array}$ & $\begin{array}{c}\text { bus } \\
3\end{array}$ & $\begin{array}{c}\text { bus } \\
4\end{array}$ & $\begin{array}{c}\text { bus } \\
5\end{array}$ & $\begin{array}{c}\text { bus } \\
6\end{array}$ & $\begin{array}{c}\text { bus } \\
7\end{array}$ & $\begin{array}{c}\text { bus } \\
8\end{array}$ & $\begin{array}{c}\text { bus } \\
9\end{array}$ & \multirow{3}{*}{$\begin{array}{c}\text { TB } \\
\text { (bus) }\end{array}$} & \multirow{3}{*}{$\begin{array}{c}\mathrm{TL} \\
(\min )\end{array}$} & \multirow{3}{*}{ do } & \multirow{3}{*}{$\begin{array}{c}f \\
\text { (bus/hour) }\end{array}$} \\
\hline & & & & \multicolumn{3}{|c|}{ tec1 } & \multicolumn{3}{|c|}{ tec2 } & \multicolumn{3}{|c|}{ tec3 } & & & & \\
\hline & & & & $\mathbf{S}$ & $\mathbf{M}$ & $\mathbf{L}$ & $\mathbf{S}$ & M & $\mathbf{L}$ & $\mathbf{S}$ & $\mathbf{M}$ & $\mathbf{L}$ & & & & \\
\hline 0 & 24.24 & 3186 & 16000 & 0 & 1311 & 170 & 0 & 85 & 0 & 0 & 0 & 0 & 1566 & 1655 & 0.540 .46 & 28.5 \\
\hline 1 & 24.26 & 2826 & 15982 & 0 & 818 & 71 & 0 & 635 & 0 & 0 & 0 & 0 & 1524 & 1588 & 0.540 .46 & 28.6 \\
\hline 2 & 24.40 & 2747 & 15989 & 0 & 593 & 96 & 0 & 731 & 72 & 0 & 0 & 0 & 1492 & 1589 & 0.530 .47 & 28.0 \\
\hline 3 & 24.44 & 2560 & 15956 & 0 & 347 & 135 & 0 & 737 & 112 & 0 & 108 & 0 & 1439 & 1483 & 0.520 .48 & 28.4 \\
\hline 4 & 24.49 & 2509 & 15967 & 0 & 303 & 0 & 0 & 1041 & 117 & 0 & 0 & 0 & 1461 & 1597 & 0.520 .48 & 27.5 \\
\hline 5 & 24.54 & 2341 & 15966 & 0 & 361 & 0 & 0 & 894 & 0 & 0 & 194 & 0 & 1449 & 1514 & 0.510 .49 & 27.7 \\
\hline 6 & 24.58 & 2228 & 15931 & 0 & 0 & 266 & 0 & 0 & 436 & 0 & 0 & 489 & 1191 & 1588 & 0.540 .46 & 22.4 \\
\hline 7 & 24.67 & 2182 & 15994 & 0 & 255 & 0 & 0 & 151 & 432 & 0 & 39 & 386 & 1263 & 1575 & 0.510 .49 & 23.8 \\
\hline 8 & 24.74 & 2005 & 15957 & 0 & 0 & 0 & 0 & 240 & 484 & 0 & 0 & 478 & 1202 & 1493 & 0.500 .50 & 23.8 \\
\hline 9 & 24.84 & 1968 & 15939 & 0 & 0 & 0 & 0 & 108 & 540 & 0 & 36 & 495 & 1179 & 1396 & 0.500 .50 & 24.7 \\
\hline 10 & 24.98 & 1689 & 15931 & 0 & 0 & 0 & 0 & 0 & 338 & 0 & 121 & 671 & 1130 & 1531 & 0.500 .50 & 22.7 \\
\hline 11 & 25.04 & 1668 & 15980 & 0 & 0 & 0 & 0 & 0 & 298 & 0 & 0 & 813 & 1111 & 1459 & 0.490 .51 & 22.5 \\
\hline 12 & 25.22 & 1423 & 15930 & 0 & 0 & 0 & 0 & 0 & 52 & 0 & 0 & 1018 & 1070 & 1400 & 0.490 .51 & 22.4 \\
\hline 13 & 25.44 & 1421 & 15918 & 0 & 0 & 0 & 0 & 0 & 51 & 0 & 0 & 1018 & 1069 & 1424 & 0.470 .53 & 21.8 \\
\hline 14 & 25.48 & 1332 & 15784 & 0 & 0 & 0 & 0 & 0 & 0 & 0 & 238 & 846 & 1084 & 1401 & 0.470 .53 & 23.1 \\
\hline
\end{tabular}

Special attention should be paid on the frequencies assigned to the lines, since, as mentioned in Section 4.3, no maximum frequency is enforced. In Table 8, it is shown that the highest average frequency is 28.6. In particular, for this solution, the frequencies range between 10 and 37 buses/h. Furthermore, in the solutions on Mumford1 without heterogeneous fleet, some frequencies are as high as $60 \mathrm{buses} / \mathrm{h}$. These 
high values, which are unrealistic in real life, are due to the relatively small bus sizes considered in this work, which were set based on the common values used in the literature for Mandl's network. Indeed, the frequencies assigned in the different solutions found for Mandl's and Mumford0 networks range between 3 and 25 buses/h, which are reasonable values. Given the scope of this paper, the high frequencies found in Mumford1 don't affect the conclusion, although more attention should be given to these values when looking for results to a specific real life instance.

\subsection{Analysis of the Local Search}

A final set of numerical experiments was performed to determine the benefits of performing the local search step and the impact of considering, during local search, the original objective or a simpler one, optimizing the proportion of direct trips as described in Section 4.2. Table 9 displays the average results of 10 runs of the MA on three instances. Three variants are considered: applying no local search (No-LS), a local search that optimizes the proportion of direct trips (LS1) and a local search that optimizes the original total travel time objective (LS2). In each case, the average best total travel time (ATT) of the solutions and the average CPU time are displayed. For clarity, also the percentage variation compared to the base case (No-LS) are presented between brackets. It can be seen that the LS1 accomplishes to improve the total travel time in the three instances with a limited impact on the CPU time compared to No-LS. Conversely, the extra improvement when applying LS2 is not clear and implies a high increase in CPU time (more than $200 \%$ in the second instance). In the smaller instances, the LS2 generates even worse solutions.

Table 9: Analysis of the Local Search.

\begin{tabular}{cccc}
\hline Network & $\begin{array}{c}\text { Local search } \\
\text { procedure }\end{array}$ & $\begin{array}{c}\text { ATT } \\
\text { (min/pass) }\end{array}$ & CPU time \\
\hline Mandl & No-LS & 11.54 & 6.1 \\
& LS1 & $11.39(-1.3 \%)$ & $6.1(+0.0 \%)$ \\
LS2 & $11.48(-0.5 \%)$ & $7.1(+25.2 \%)$ \\
Mumford0 & No-LS & 18.07 & 68.6 \\
& LS1 & $17.53(-3.0 \%)$ & $70.0(+2.0 \%)$ \\
LS2 & $17.67(-2.2 \%)$ & $221.6(+223.0 \%)$ \\
& No-LS & 25.12 & 444.4 \\
& LS1 & $24.47(-2.6 \%)$ & $553.8(+24.6 \%)$ \\
& LS2 & $24.46(-2.6 \%)$ & $830.0(+86.8 \%)$ \\
\hline
\end{tabular}

\section{Conclusions and future work}

In this paper, the Transit Network Design and Frequency Setting Problem is addressed by means of a biobjective memetic algorithm. The two objectives considered are the minimization of the total travel time 
of the passengers through the network and the minimization of the $\mathrm{CO}_{2}$ emissions generated by all the buses in the system. The operator's point of view is considered as a budget constraint limiting the operator cost. Furthermore, a heterogeneous fleet is considered such that buses of different sizes and technologies may be assigned to the proposed lines. The memetic algorithm constructs a population of solutions that evolves through successive generations by means of crossover and mutation operators. A local search procedure is incorporated in order to improve the quality of the solutions generated. In our approach, crossover and mutation operators, as well as the local search procedure, deal mainly with the lines' configuration. The frequencies and bus sizes are selected during the evaluation procedure. This evaluation procedure corresponds to solving the transit assignment sub-problem and is addressed with a frequency share based heuristic that we modified to include the bus type selection. Our algorithm is based on the NSGA-II framework to deal with the two objectives and since it works with a population of solutions, it provides in each run a set of non-dominated solutions that allow to explore the trade-off between the two conflicting objectives. It is then up to the decision-maker to choose the compromise solution.

The experimental results on five benchmark instances demonstrate that the proposed algorithm performs well, obtaining solutions with similar quality as the ones reported in the state of the art, with reasonable computing times. A limitation of the MA compared to the literature is the constraint of accepting at most one transfer. More transfers can easily be considered in the algorithm, but for larger instances this becomes very time consuming. Moreover, the results demonstrated that very good solutions are found even with this assumption. Furthermore, the results show the benefits of both considering the $\mathrm{CO}_{2}$ emissions as well as assigning a heterogeneous fleet during network design. Significant reductions in the emission can be achieved while only slightly increasing the total travel time and maintaining the cost. In the instances tested, the emissions from the solution with the minimum travel time can be reduced around $30 \%$ by increasing the travel time only around $1.5 \%$. Even in the scenario with a homogeneous fleet the emissions can be reduced by almost $10 \%$ by increasing the travel time $1 \%$. Those results show the convenience of considering $\mathrm{CO}_{2}$ emissions and a heterogeneous fleet during the network design stage in order to design efficient public transport systems.

A future research path would be to improve the frequency setting and bus selection procedures so that they take into consideration the objectives explicitly, since now they only focus on generating feasible solutions. This would probably lead to better line plans and frequencies. Moreover, if this would be required for addressing larger networks, the computation time could be reduced by implementing parallelization. The structure of our MA should allow for parallelization without many issues. Finally, in order to bring this problem closer to practice, specific constraints in the operation of some bus technologies and more sophisticated emission models should be considered. More pollutants may be 
considered besides $\mathrm{CO}_{2}$, such as $\mathrm{NO}_{\mathrm{x}}, \mathrm{PM}$ or VOC, which have impacts not only on the environment but also on human health. Additionally, models that consider that the emission factor of a bus varies depending on speed or road conditions may be used. As a result, the emissions can no longer be modelled with a simple linear relation to the time or distance travelled by each bus. This would probably also require more advanced techniques to deal with these models while solving the bi-objective Transit Network Design and Frequency Setting Problem with emissions.

\section{Acknowledgment}

This work was supported by CONICYT (Chilean National Commission for Scientific and Technological Research) "Becas Chile" 72180156 and the FWO (Research Foundation Flanders) project G.0853.16N.

\section{References}

Afandizadeh, S., Khaksar, H., Kalantari, N., 2013. Bus fleet optimization using genetic algorithm a case study of Mashhad. Int. J. Civ. Eng. 11, 43-52.

Ahmed, L., Mumford, C., Kheiri, A., 2018. Solving urban transit route design problem using selection hyper-heuristics. Eur. J. Oper. Res. https://doi.org/10.1016/j.ejor.2018.10.022

An, K., Lo, H.K., 2016. Two-phase stochastic program for transit network design under demand uncertainty. Transp. Res. Part B Methodol. 84, 157-181. https://doi.org/10.1016/j.trb.2015.12.009

Arbex, R.O., da Cunha, C.B., 2015. Efficient transit network design and frequencies setting multiobjective optimization by alternating objective genetic algorithm. Transp. Res. Part B Methodol., Optimization of Urban Transportation Service Networks 81, 355-376. https://doi.org/10.1016/j.trb.2015.06.014

Asadi Bagloee, S., Ceder, A. (Avi), 2011. Transit-network design methodology for actual-size road networks. Transp. Res. Part B Methodol. 45, 1787-1804. https://doi.org/10.1016/j.trb.2011.07.005

Baaj, M.H., Mahmassani, H., 1990. TRUST: A LISP program for the analysis of transit route configurations. Transp. Res. Rec. 1283, 125-135.

Baaj, M.H., Mahmassani, H.S., 1991. An Al-based approach for transit route system planning and design. J. Adv. Transp. 25, 187-209. https://doi.org/10.1002/atr.5670250205

Beltran, B., Carrese, S., Cipriani, E., Petrelli, M., 2009. Transit network design with allocation of green vehicles: A genetic algorithm approach. Transp. Res. Part C Emerg. Technol., Artificial Intelligence in Transportation Analysis: Approaches, Methods, and Applications 17, 475-483. https://doi.org/10.1016/j.trc.2009.04.008

Blum, J.J., Mathew, T.V., 2011. Intelligent Agent Optimization of Urban Bus Transit System Design. J. Comput. Civ. Eng. 25, 357-369. https://doi.org/10.1061/(ASCE)CP.19435487.0000095

Borndörfer, R., Grötschel, M., Pfetsch, M.E., 2007. A Column-Generation Approach to Line Planning in Public Transport. Transp. Sci. 41, 123-132. https://doi.org/10.1287/trsc.1060.0161 
Buba, A.T., Lee, L.S., 2018. A differential evolution for simultaneous transit network design and frequency setting problem. Expert Syst. Appl. 106, 277-289. https://doi.org/10.1016/j.eswa.2018.04.011

Canca, D., De-Los-Santos, A., Laporte, G., Mesa, J.A., 2017. An adaptive neighborhood search metaheuristic for the integrated railway rapid transit network design and line planning problem. Comput. Oper. Res. 78, 1-14. https://doi.org/10.1016/j.cor.2016.08.008

Cancela, H., Mauttone, A., Urquhart, M.E., 2015. Mathematical programming formulations for transit network design. Transp. Res. Part B Methodol. 77, 17-37. https://doi.org/10.1016/j.trb.2015.03.006

Ceder, A., Wilson, N.H.M., 1986. Bus network design. Transp. Res. Part B Methodol. 20, 331-344. https://doi.org/10.1016/0191-2615(86)90047-0

Chakroborty, P., Wivedi, T., 2002. Optimal Route Network Design for Transit Systems Using Genetic Algorithms. Eng. Optim. 34, 83-100. https://doi.org/10.1080/03052150210909

Cipriani, E., Gori, S., Petrelli, M., 2012. Transit network design: A procedure and an application to a large urban area. Transp. Res. Part C Emerg. Technol., Special issue on Optimization in Public Transport+ISTT2011 20, 3-14. https://doi.org/10.1016/j.trc.2010.09.003

Deb, K., Pratap, A., Agarwal, S., Meyarivan, T., 2002. A fast and elitist multiobjective genetic algorithm: NSGA-II. IEEE Trans. Evol. Comput. 6, 182-197. https://doi.org/10.1109/4235.996017

Duran, J., Pradenas, L., Parada, V., 2019. Transit network design with pollution minimization. Public Transp. 11, 189-210. https://doi.org/10.1007/s12469-019-00200-5

Ercan, T., Zhao, Y., Tatari, O., Pazour, J.A., 2015. Optimization of transit bus fleet's life cycle assessment impacts with alternative fuel options. Energy 93, 323-334. https://doi.org/10.1016/j.energy.2015.09.018

Fan, L., Mumford, C.L., Evans, D., 2009. A simple multi-objective optimization algorithm for the urban transit routing problem, in: 2009 IEEE Congress on Evolutionary Computation. Presented at the 2009 IEEE Congress on Evolutionary Computation, pp. 1-7. https://doi.org/10.1109/CEC.2009.4982923

Farahani, R.Z., Miandoabchi, E., Szeto, W.Y., Rashidi, H., 2013. A review of urban transportation network design problems. Eur. J. Oper. Res. 229, 281-302. https://doi.org/10.1016/j.ejor.2013.01.001

Feng, X., Zhu, X., Qian, X., Jie, Y., Ma, F., Niu, X., 2019. A new transit network design study in consideration of transfer time composition. Transp. Res. Part D Transp. Environ., Special Issue on Electromobility for Green Transportation Systems and Sustainable Environment 66, 85-94. https://doi.org/10.1016/j.trd.2018.03.019

Goerigk, M., Schmidt, M., 2017. Line planning with user-optimal route choice. Eur. J. Oper. Res. 259, 424-436. https://doi.org/10.1016/j.ejor.2016.10.034

Guihaire, V., Hao, J.-K., 2008. Transit network design and scheduling: A global review. Transp. Res. Part A Policy Pract. 42, 1251-1273. https://doi.org/10.1016/j.tra.2008.03.011

Häll, C.H., Ceder, A. (Avi), Ekström, J., Quttineh, N.-H., 2019. Adjustments of public transit operations planning process for the use of electric buses. J. Intell. Transp. Syst. 23, 216230. https://doi.org/10.1080/15472450.2018.1488131 
Iliopoulou, C., Tassopoulos, I., Kepaptsoglou, K., Beligiannis, G., 2019. Electric Transit Route Network Design Problem: Model and Application. Transp. Res. Rec. 0361198119838513. https://doi.org/10.1177/0361198119838513

Islam, K.A., Moosa, I.M., Mobin, J., Nayeem, M.A., Rahman, M.S., 2019. A heuristic aided Stochastic Beam Search algorithm for solving the transit network design problem. Swarm Evol. Comput. 46, 154-170. https://doi.org/10.1016/j.swevo.2019.02.007

Jha, S.B., Jha, J.K., Tiwari, M.K., 2019. A multi-objective meta-heuristic approach for transit network design and frequency setting problem in a bus transit system. Comput. Ind. Eng. 130, 166-186. https://doi.org/10.1016/j.cie.2019.02.025

John, M.P., Mumford, C.L., Lewis, R., 2014. An Improved Multi-objective Algorithm for the Urban Transit Routing Problem, in: Evolutionary Computation in Combinatorial Optimisation, Lecture Notes in Computer Science. Presented at the European Conference on Evolutionary Computation in Combinatorial Optimization, Springer, Berlin, Heidelberg, pp. 49-60. https://doi.org/10.1007/978-3-662-44320-0_5

Kay, M., Clark, M., Duffy, C., Laube, M., Lian, F., 2011. Bus Lifecycle Cost Model for Federal Land Management Agencies: User's guide. John A. Volpe National Transportation Systems Center, Cambridge, Massachusetts.

Kechagiopoulos, P.N., Beligiannis, G.N., 2014. Solving the Urban Transit Routing Problem using a particle swarm optimization based algorithm. Appl. Soft Comput. 21, 654-676. https://doi.org/10.1016/j.asoc.2014.04.005

Kepaptsoglou, K., Karlaftis, M., 2009. Transit Route Network Design Problem: Review. J. Transp. Eng. 135, 491-505. https://doi.org/10.1061/(ASCE)0733-947X(2009)135:8(491)

Lee, Y.-J., Vuchic, V.R., 2005. Transit Network Design with Variable Demand. J. Transp. Eng. 131, 1-10. https://doi.org/10.1061/(ASCE)0733-947X(2005)131:1(1)

Liu, L., Olszewski, P., Goh, P.-C., 2010. Combined Simulated Annealing and Genetic Algorithm Approach to Bus Network Design, in: Transport Systems Telematics, Communications in Computer and Information Science. Presented at the International Conference on Transport Systems Telematics, Springer, Berlin, Heidelberg, pp. 335-346. https://doi.org/10.1007/978-3-642-16472-9_37

López-Ramos, F., 2014. Integrating network design and frequency setting in public transportation networks: a survey. SORT-Stat. Oper. Res. Trans. 38, 181-214.

Mahdavi Moghaddam, S.H., Rao, K.R., Tiwari, G., Biyani, P., 2019. Simultaneous Bus Transit Route Network and Frequency Setting Search Algorithm. J. Transp. Eng. Part A Syst. 145, 04019011. https://doi.org/10.1061/JTEPBS.0000229

Mandl, C.E., 1979. Applied network optimization. Academic Press, London ; New York.

Mauttone, A., Urquhart, M.E., 2009. A multi-objective metaheuristic approach for the Transit Network Design Problem. Public Transp. 1, 253-273. https://doi.org/10.1007/s12469010-0016-7

Moscato, P., 1989. On Evolution, Search, Optimization, Genetic Algorithms and Martial Arts Towards Memetic Algorithms (C3P Report 826). Tech. Rep. Calif. Inst. Technol.

Mumford, C.L., 2013. New heuristic and evolutionary operators for the multi-objective urban transit routing problem, in: 2013 IEEE Congress on Evolutionary Computation. Presented at the 2013 IEEE Congress on Evolutionary Computation, pp. 939-946. https://doi.org/10.1109/CEC.2013.6557668 
Nayeem, M.A., Rahman, M.K., Rahman, M.S., 2014. Transit network design by genetic algorithm with elitism. Transp. Res. Part C Emerg. Technol. 46, 30-45. https://doi.org/10.1016/j.trc.2014.05.002

Ngamchai, S., Lovell, D., 2003. Optimal Time Transfer in Bus Transit Route Network Design Using a Genetic Algorithm. J. Transp. Eng. 129, 510-521. https://doi.org/10.1061/(ASCE)0733947X(2003)129:5(510)

Nikolić, M., Teodorović, D., 2014. A simultaneous transit network design and frequency setting: Computing with bees. Expert Syst. Appl. 41, 7200-7209. https://doi.org/10.1016/j.eswa.2014.05.034

Owais, M., Osman, M.K., 2018. Complete hierarchical multi-objective genetic algorithm for transit network design problem. Expert Syst. Appl. 114, 143-154. https://doi.org/10.1016/j.eswa.2018.07.033

Pternea, M., Kepaptsoglou, K., Karlaftis, M.G., 2015. Sustainable urban transit network design. Transp. Res. Part A Policy Pract. 77, 276-291. https://doi.org/10.1016/j.tra.2015.04.024

Schöbel, A., Scholl, S., 2006. Line Planning with Minimal Traveling Time, in: Kroon, L.G., Möhring, R.H. (Eds.), 5th Workshop on Algorithmic Methods and Models for Optimization of Railways (ATMOS'05), OpenAccess Series in Informatics (OASIcs). Schloss DagstuhlLeibniz-Zentrum fuer Informatik, Dagstuhl, Germany. https://doi.org/10.4230/OASIcs.ATMOS.2005.660

Szeto, W.Y., Jiang, Y., 2014. Transit route and frequency design: Bi-level modeling and hybrid artificial bee colony algorithm approach. Transp. Res. Part B Methodol. 67, 235-263. https://doi.org/10.1016/j.trb.2014.05.008

Talbi, E.-G., 2009. Metaheuristics: From Design to Implementation. John Wiley \& Sons, Hoboken, New Jersey.

Ul Abedin Zain, Busch Fritz, Wang David Z. W., Rau Andreas, Du Bo, 2018. Comparison of Public Transport Network Design Methodologies Using Solution-Quality Evaluation. J. Transp. Eng. Part A Syst. 144, 04018036. https://doi.org/10.1061/JTEPBS.0000159

Wan, Q.K., Lo, H.K., 2009. Congested multimodal transit network design. Public Transp. 1, 233251. https://doi.org/10.1007/s12469-009-0015-8

Yan, Y., Liu, Z., Meng, Q., Jiang, Y., 2013. Robust Optimization Model of Bus Transit Network Design with Stochastic Travel Time. J. Transp. Eng. 139, 625-634. https://doi.org/10.1061/(ASCE)TE.1943-5436.0000536

Yen, J.Y., 1971. Finding the K Shortest Loopless Paths in a Network. Manag. Sci. 17, 712-716.

Zhang, L., Yang, H., Wu, D., Wang, D., 2014. Solving a discrete multimodal transportation network design problem. Transp. Res. Part C Emerg. Technol. 49, 73-86. https://doi.org/10.1016/j.trc.2014.10.008

Zhao, H., Xu, W., Jiang, R., 2015. The Memetic algorithm for the optimization of urban transit network. Expert Syst. Appl. 42, 3760-3773. https://doi.org/10.1016/j.eswa.2014.11.056 